\title{
Intergroup Rhabdomyosarcoma Group IIc
}

National Cancer Institute

\section{Source}

National Cancer Institute. Intergroup Rhabdomyosarcoma Group IIc. NCI Thesaurus.

Code C148018.

Regional disease with positive lymph nodes. 